\title{
An Estimation Method of Fire Extinguishing Time in Closed Ship Cabin
}

\author{
Tang Fang ${ }^{1, \mathrm{a}}$, Li Pan ${ }^{1, \mathrm{~b}}$, Chen Guofeng ${ }^{1, \mathrm{c}}$, Xie Jianghui ${ }^{1, \mathrm{~d}}$ \\ ${ }^{1}$ Wuhan Second Ship Design and Research Institute, Wuhan, 430205, China
}

\begin{abstract}
There are many potential fire hazards in ship engine cabin, especially in the closed environment of submarine underwater. Fires in cramped cabin are usually harder to put out, therefore, enclosing cabin is a possible means of extinguishing fire. In this paper, typical fire scene in ship engine cabin is analyzed firstly, then a two-layer zone model of engine cabin fire is established combined with structural characteristics of engine cabin. According to the mass conservation and energy conservation, fire smoke layer change rule is described to estimate extinguishing time. At last, typical hydraulic jet fire and electrical fire smoke layer calculation is taken as an example. The result shows that enclosing cabin is a fast and effective method, and the method of estimating fire extinguishing time in engine cabin by this paper is feasible.
\end{abstract}

\section{Introduction}

In order to carry out covert combat missions, the modern submarine needs to sail in great depth in a long time. Complex mechanical-electrical equipment and system is set in internal cabin, especially in the engine cabin, which is full of high pressure hydraulic oil, lubricating oil, diesel oil and high voltage electrical equipment and other potential hazards. Smoke generated by fire event may fill in the cabin and cause loss of power, sinking or other serious consequences, therefore, engine fire risk is high. According to statistics of 30 years after the second world war, the former Soviet Union has occurred 16 major accidents, among which fire-related accidents accounted for $62.5 \%$ and $62 \%$ of the deaths[1]. Due to the limitation of underwater closed environment, it is difficult to extinguish fire in the narrow cabin, so adopting the measures of enclosing cabin to extinguish serious fire is necessary and possible. Enclosing cabin means stop all non-critical equipment or systems, close all vents, and make use of the features of cabin structure to allow the fire to burn in the airtight cabin. Until oxygen is insufficient, the fire will be extinguished automatically and achieve the purpose of extinguishing fire[2].Based on large eddy simulation of FDS, fire dynamic characteristics in the closed cabin of ship are studied[3-5]. The ship cabin space is divided into many grids to calculate heat exchange between them. The more grids are, the greater amount of calculation is. In this paper, the two-layer zone model of ship engine cabin fire is adopted to establish the change rule of cabin fire smoke layer height, and then the estimation method of enclosing cabin fire extinguishing time is obtained.

\section{Ship engine cabin fire scene}

There are a large number of electrical and other equipment in the ship engine cabin. The electrical equipment has many potential fire hazards, such as cables, relays and circuit boards. Due to the reduction of insulation cause by humidity environment, internal short circuit, overheating and other factors may directly lead electrical equipment fire. Finland VTT technical research center has carried out electrical cabinets fire experiment, the test electrical cabinet size is $580 \mathrm{~mm} \times 600 \mathrm{~mm} \times 2250 \mathrm{~mm}$, which has configured different quality of circuit boards, relays and cable inside (fuel quality is $60 \sim 90 \mathrm{~kg}$ ), conducted a series of combustion test to obtain fire heat release rate(HRR) under different configuration, the peak heat release rate(PHRR) is about $100 \mathrm{~kW}$ to $400 \mathrm{~kW}[6]$.

There are also a large number of high-pressure hydraulic oil and other oil pipelines in the engine cabin. When these pipelines accidentally leak, the high-pressure oil will become spray state which is easy to burn or explode. For example, former Soviet Union submarine "communist youth league" was on its way back to the base after mission in 1989, a fire suddenly broke out in cabin 7, which quickly spread to cabin 6 and caused hydraulic device oil leak of turbine engine. Then a hydraulic spray fire was made which spread continuously and finally resulted in a major sinking accident [7]. The leakage amount of hydraulic oil is determined by flow coefficient, pressure and density. The greater leakage flow, the greater HRR is. The US navy had conducted a fire risk experiment for leakage of hydraulic system. During the experiment, the pressure of hydraulic system was 10MPa, and HRR of hydraulic spray fire was 
$1.26 \mathrm{MW}$ [8]. It can be seen that hydraulic spray fire is much more powerful than electrical fire, and more heat is generated in a short time.

\section{Two-layer zone model of ship engine cabin fire}

The engine cabin is a power system cabin which locates many large pieces of equipment and connects together without any entity separation. The smoke generated by fire can accumulate freely from top of the engine cabin, and then gradually spread downward. The development process of engine cabin fire can be described by two-layer zone model. The two-layer zone model is based on experimental observation and can be used to predict the fire development process[9]. This paper suppose the engine cabin is a cylinder structure, after fire, hot smoke accumulates to the top of cabin(Figure 1), and cabin space is divided into two zones: top smoke (top shadow in Figure 1) and bottom air (bottom layer in Figure 1). The fire plume transmits momentum, heat, and mass, therefore, a set of ordinary differential equations can be established according to the principles of mass conservation, energy conservation, law of thermodynamics and so on.

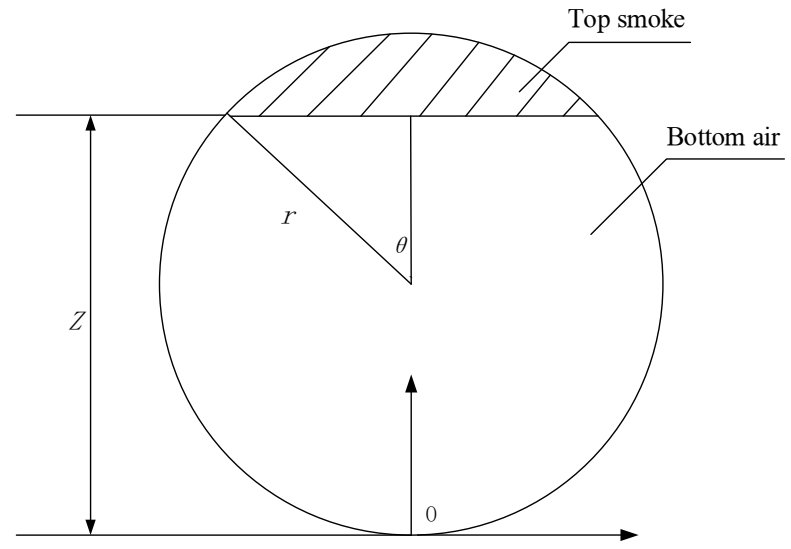

Fig.1. smoke of ship engine cabin fire

In order to establish a mathematical zone model of engine cabin fire, the following assumptions are first made:

(1) Physical parameters in each zone are uniform;

(2) The hatch cover of engine cabin is closed, only some air leaks into the cabin through the gap of hatch cover;

(3) During the whole fire process, air pressure in the cabin is constant, meanwhile, air and smoke is regarded as ideal gases with the same molecular weight;

(4) All fuel can burn continuously until fire has extinguished.

According to the mass conservation of bottom layer air, the following relation can be obtained:

$$
m_{L}+m_{p}+m_{e}=0
$$

Where, $m_{L}$ is the change of bottom layer air quality, $m_{p}$ is the plume flow, $m_{e}$ is the flow of leaking gas in the cabin.

The change relationship of bottom layer air quality can be expressed by the following formula:

$$
m_{L}=\rho_{L} \frac{d V}{d t}
$$

Where, $\rho_{L}$ is the density of bottom layer air, $V$ is the volume of top layer smoke.

The volume of top layer smoke can be expressed by the following formula:

$$
\begin{gathered}
V=l S_{\text {smoke }}=l\left(r^{2} \arccos \theta-r^{2} \cos \theta \sin \theta\right) \\
Z=r+r \cos \theta
\end{gathered}
$$

Where, $Z$ is the height of top layer smoke, $r$ is the radius of engine cabin, and $l$ is the length of engine cabin.

According to the point source plume flow relationship proposed by Zukoski[10], $m_{p}$ can be represented by the following formula:

$$
m_{P}=0.21\left[\frac{\left(1-L_{r}\right) Q g}{C_{p} T_{L}}\right]^{1 / 3} \rho_{L}^{2 / 3} Z^{5 / 3}
$$

Where, $L_{r}$ is the radiant heat loss coefficient, $g$ is the gravitational acceleration, $C_{P}$ is the specific heat at constant pressure of air, $T_{L}$ is the temperature of bottom layer, $Q$ is the HRR of the ignition source.

According to the conservation of energy in engine cabin, the heat released by fire source to cabin air is equal to the energy loss of internal air, which can be represented by the following formula:

$$
\left(1-L_{c}\right) Q=m_{e} C_{p} T_{L}
$$

Where, $L_{c}$ is the total heat loss coefficient.

By substituting the above equation (2) (6) into equation (1), we can obtain following equation:

$$
\rho_{L} \frac{d\left[\left(r^{2} \arccos \left(\frac{Z-r}{r}\right)-r^{2} \frac{Z-r}{r} \sqrt{\left.\left.1-\left(\frac{Z-r}{r}\right)^{2}\right)\right]}\right.\right.}{d t}+0.21\left[\frac{\left(1-L_{L}\right) Q g}{C_{P} T_{L}}\right]^{1 / 3} \rho_{L}^{2 / 3} Z^{5 / 3}+\frac{\left(1-L_{L}\right) Q}{C_{\rho} T_{L}}=0
$$

assume that:

$$
\frac{Z-r}{r}=Y
$$

thus, equation (7) is shown as:

$$
\rho_{L} l r^{2}\left(-\frac{1}{\sqrt{1-Y^{2}}}-\sqrt{1-Y^{2}}+\frac{Y^{2}}{\sqrt{1-Y^{2}}}\right) \frac{d Y}{d t}+0.21\left[\frac{\left(1-L_{r}\right) Q_{g}}{C_{p} T_{L}}\right]^{1 / 3} \rho_{L}^{2 / 3}(r Y+r)^{5 / 3}+\frac{\left(1-L_{c}\right) Q}{C_{p} T_{L}}=0
$$

By solving equation (9), we can obtain change relation of smoke layer height $Z$ with time duration.

When $Z$ becomes 0 , the air inside cabin is considered to be consumed, and this time is the extinguishing time of enclosing cabin.

\section{Calculation examples of enclosing cabin to extinguish fire}

Suppose a engine cabin is a cylinder, radius $r=4 \mathrm{~m}$, length $l=8 \mathrm{~m}$, radiant heat loss coefficient $L_{r}=0.35$, total heat loss coefficient $L_{c}=0.35$, specific heat at constant pressure $C_{P}=1004 \mathrm{~J} /(\mathrm{kg} \cdot \mathrm{K})$, bottom air temperature $T_{L}=$ $300 \mathrm{~K}$, bottom air density $\rho_{L}=1.19 \mathrm{~kg} / \mathrm{m}^{3}$, gravity acceleration $g=9.8 \mathrm{~m} / \mathrm{s}^{2}$. Matlab/Simulink is used to set up simulation model and calculate height of smoke layer $Z$ according to equation (9).

According to hydraulic leakage fire test results combined with the United States Navy, and assuming 
that engine cabin hydraulic system working pressure is $5 \mathrm{MPa}$, therefore, the hydraulic spray fire $\mathrm{HRR} Q=$ $0.89 \mathrm{MW}$. The smoke layer height $Z$ changing curve is shown in Figure 2, where, $\mathrm{X}$ axis is time duration, $\mathrm{Y}$ axis is smoke layer height.

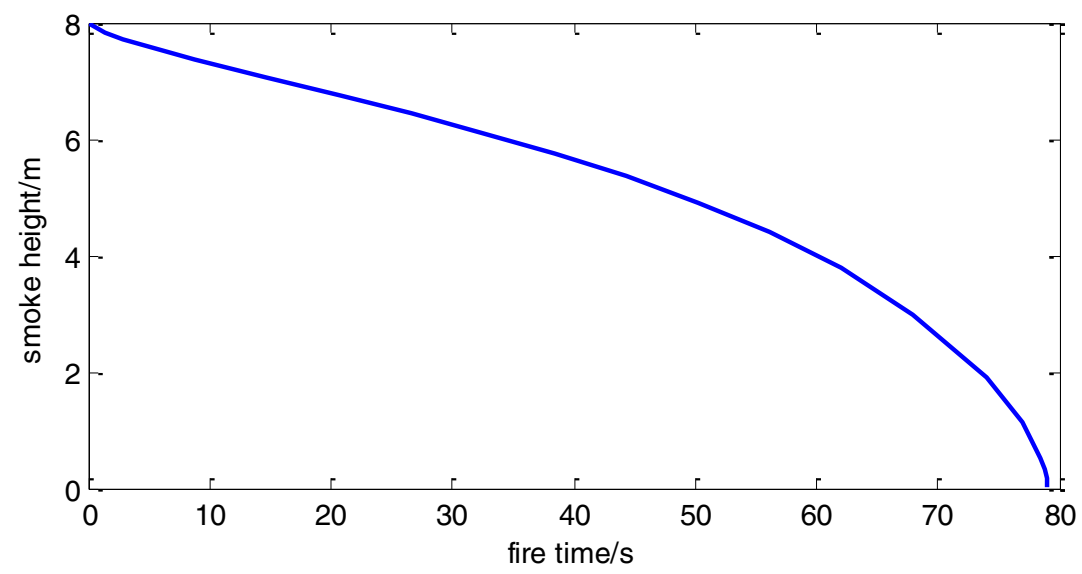

Fig.2. Hydraulic spray fire smoke layer height change

It is assumed that HRR of electrical cabinet fire in the engine cabin is $Q=100 \mathrm{~kW}$, based on the test results of
VTT technical research center in Finland. The smoke layer height $Z$ changing curve is shown in Figure 3.

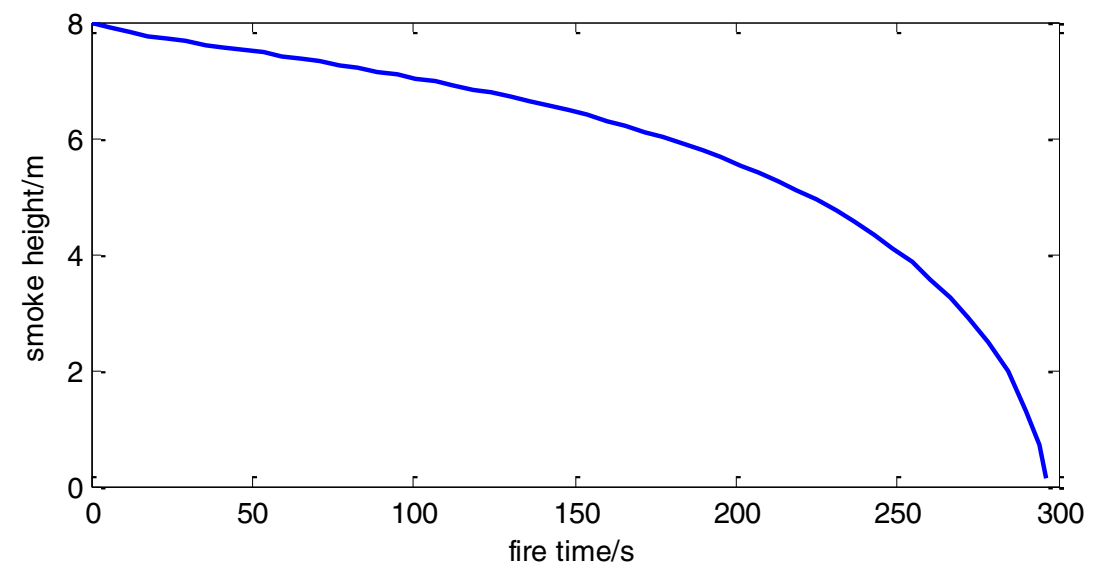

Fig.3. Electrical fire smoke layer height change

It can be seen from above calculation results that smoke layer height of hypothetical electrical fire changes to 0 in about $296 \mathrm{~s}$, so that we can consider fire extinguishing time by enclosing cabin is $296 \mathrm{~s}$. The smoke layer height of hydraulic spray fire changes to 0 in about 80 s, so that we can consider fire extinguishing time by enclosing cabin is 80 s. Comparing to electrical fire, HRR of hydraulic spray fire is much bigger, and is faster to extinguish by enclosing cabin since oxygen consumes more quickly.

\section{Conclusions}

By analyzing fire scenes and fire development characteristics of ship engine cabin, this paper has established a mathematical model to estimate fire extinguishing time of engine cabin by using two-layer zone model. Then simulated hypothetical hydraulic spray fire and electrical fire by Matlab/Simulink is calculated to obtain fire extinguishing time of engine cabin. The calculated result indicates that enclosing cabin to extinguish fire is a fast and effective method.
The fire extinguishing time estimated by this paper is based on total consumption of bottom air layer, but actually it may be extinguished when oxygen reduces to a certain extent. In the next step, the calculation model can be further improved in combination with consumption of oxygen.

\section{References}

1. Lin Fanca, Liu Baishun, Hao Yinze, Submarine fire accident rules and fire prevention measures, Proceedings of ship pollution prevention management, 2008:117-119

2. Li Ying, Pu Jinyun, Chen Xiaohong, Application of water-mist fire suppression to submarine's ablaze airtight cabin, Jouranl of PLA university of science and technology, 2011,21(4):378-382

3. Sun Shifang, QIN Guangyao, Liu Bo, The Analysis of Cabin Ship Fire Safety, Journal of zhejiang Institute of Communications, 2012,13(3):36-40 
4. Su Shichuan, Wang Liang, Nie Yuhong, Gu Xiang, Numerical simulation and strategy analysis of fire development process in a certain ship engine room, Fire Science and Technology, 2019, 28(1):15-19

5. Zhang Boshi, Lu Shouxiang, Analysis of the Influence of Mechanical Ventilation on Smoke Control during the Ship Engine Room Fire, Ship and Ocean Engineering, 2013,42(4):31-34

6. J.Mangs, J.Paananen, O.Keski-Rahkonen, Calorimetric fire experiment on electronic cabinets, fire safety journal, 38(2013):165-186

7. $\mathrm{Gu}$ Huihe, The fire buried the Communist youth league nuclear submarine, Marine Fire, 2008(6):46-47

8. Yu Tao, Tian Wan, Zhi Pin, Review on US Submarine Hydraulic System Explosion and Fire Hazards, Shipping engineering, 2015(1):6-9

9. Friedman. Survey of computer models for fire and smoke, Forum for International Cooperation on Fire Research, Factory Mutual Research Corporation, Norwood, Ma02062, U.S.A. 1991

10. Cetegen B M, Zukoski E E, Kubota T, Entrainment and flame geometry of fire plumes, USA: California Institute Technology, 1982. 